\title{
DECOMPOSIÇÃO TÉRMICA DO BICARBONATO DE SÓDIO - DO PROCESSO SOLVAY AO DIAGRAMA TIPO ELLINGHAM
}

\author{
Alessandra de Souza Maia e Viktoria Klara Lakatos Osorio* \\ Instituto de Química, Universidade de São Paulo, CP 26077, 05513-970 São Paulo - SP
}

Recebido em 29/7/02; aceito em 5/12/02

\begin{abstract}
THERMAL DECOMPOSITION OF SODIUM BICARBONATE - FROM SOLVAY PROCESS TO AN ELLINGHAM TYPE DIAGRAM. A didactic experiment based on the thermal decomposition of sodium bicarbonate using a reagent found in the marketplace is proposed. The reaction products are identified by qualitative tests and stoichiometric calculations. The thermal stability of carbonates and the influence of lattice energies are discussed, emphasizing periodic trends in the alkali and alkaline earth families. The industrial importance of the reaction is also explored.
\end{abstract}

Keywords: sodium bicarbonate; Ellingham type diagrams; Solvay process.

\section{INTRODUÇÃO}

Uma grande parcela dos alunos de diversas carreiras, que cursaram disciplinas introdutórias de Química no Instituto de Química da USP, tiveram em suas aulas de laboratório um experimento sobre decomposição térmica de bicarbonato de potássio ${ }^{1}$. Ele foi proposto objetivando exercitar cálculos estequiométricos e introduzir a técnica de pesagem em balança analítica, utilizada para determinar a massa do bicarbonato inicial e a massa do sólido obtido após o aquecimento. A equação do processo era fornecida para os alunos.

Com a contribuição de docentes que ministraram essas disciplinas, o experimento foi sendo aprimorado. Foram incorporados testes qualitativos para identificar os produtos da decomposição, que consistem de um gás e um sólido. O experimento passou então a ilustrar também tipos de reações químicas, tais como ácido-base, precipitação e complexação. Com o número sempre crescente de alunos, a balança analítica foi substituída por balança semi-analítica, porém o sistema investigado continuou o mesmo: bicarbonato de potássio.

A identificação do produto sólido é o ponto mais instigante da investigação, necessária para que os alunos possam propor a equação química representativa da decomposição térmica. A expectativa dos alunos com relação a esse produto usualmente não corresponde à realidade. Isto mostra que o experimento se presta a uma discussão mais ampla, no âmbito da Química Inorgânica, ilustrando conceitos relacionados com ligações iônicas, caráter ácido-base e variação de funções termodinâmicas com a temperatura. $\mathrm{O}$ presente artigo explora estas possibilidades, ao mesmo tempo em que propõe a substituição do bicarbonato de potássio pelo de sódioº ${ }^{2}$. A decomposição térmica do bicarbonato de sódio apresenta importância industrial, por ser a última etapa do processo Solvay de fabricação de soda ${ }^{3-5}$. Além disso, bicarbonato de sódio pode ser adquirido em farmácias e supermercados, a um custo bem inferior ao de reagentes especiais para uso em laboratórios. O termo bicarbonato é utilizado para designar, no comércio, o ânion hidrogenocarbonato, enquanto soda e soda cáustica se referem a carbonato de sódio e hidróxido de sódio, respectivamente.

*e-mail: vklosori@iq.usp.br

\section{PARTE EXPERIMENTAL}

\section{Estudo quantitativo da decomposição térmica do bicarbonato} de sódio

Pesa-se um tubo de ensaio seco, provido de rolha de borracha, utilizando uma balança semi-analítica, com legibilidade de centésimos de grama. A seguir, adicionam-se aproximadamente $2 \mathrm{~g}$ de bicarbonato de sódio ao tubo, adapta-se a rolha e pesa-se novamente o conjunto.

Remove-se a rolha e, com o auxílio de uma pinça, aquece-se o tubo na chama do bico de Bünsen, suave e intermitentemente no início e depois diretamente, durante 3 a 4 min. Leva-se o tubo para uma estufa a cerca de $100{ }^{\circ} \mathrm{C}$, durante $10 \mathrm{~min}$. A seguir, transfere-se o tubo para um dessecador, deixando-o atingir a temperatura ambiente.

Tampa-se o tubo com a mesma rolha usada anteriormente e pesase o conjunto.

Identificação da fase gasosa formada na decomposição térmica do bicarbonato de sódio

Umedece-se uma tira de papel de filtro com uma solução $1 \mathrm{~mol} \mathrm{~L}^{-1}$ de cloreto de cobalto(II) e seca-se o papel na estufa. Adiciona-se bicarbonato de sódio sólido a um tubo de ensaio seco e adapta-se a tira de papel de filtro, recentemente retirada da estufa, na parte superior do tubo. Aquece-se o bicarbonato diretamente na chama do bico de Bünsen e observa-se o que ocorre com o papel na parte fria, superior, do tubo de ensaio.

Utilizando um sistema fechado, conforme recomendado nos textos didáticos para análise qualitativa de carbonatos $^{6}$, aquece-se bicarbonato de sódio sólido num tubo de ensaio e recolhe-se o gás formado em uma solução $0,2 \mathrm{~mol} \mathrm{~L}^{-1}$ de hidróxido de bário.

\section{Identificação do produto sólido da decomposição térmica do bicarbonato de sódio}

Utilizando ainda o sistema fechado, coloca-se, num dos tubos de ensaio, o produto sólido obtido no estudo quantitativo efetuado. Adiciona-se ao sólido solução $1 \mathrm{~mol} \mathrm{~L}^{-1}$ de ácido clorídrico, fecha-se o tubo rapidamente e aquece-se o mesmo, recolhendo o gás formado em uma solução de hidróxido de bário. 


\section{RESULTADOS E DISCUSSÃO}

A equação representativa da decomposição térmica é deduzida após serem identificados os produtos gasosos e o sólido. $\mathrm{Na}$ fase gasosa, identificam-se $\mathrm{CO}_{2}$ e $\mathrm{H}_{2} \mathrm{O}$, o primeiro pela reação com solução de $\mathrm{Ba}(\mathrm{OH})_{2}$ precipitando $\mathrm{BaCO}_{3}$ e a segunda pela ação sobre cloreto de cobalto anidro. Este teste em geral já é familiar aos alunos, em virtude do uso como indicador de umidade em sílica-gel e em objetos (galinhos, bailarinas, etc.) encontrados no comércio para previsão do tempo e também como "tinta mágica", revelada por aquecimento $^{7,8}$. Baseia-se na mudança de cor decorrente da conversão entre a forma anidra, $\mathrm{CoCl}_{2}$, azul, e o composto hidratado, $\left[\mathrm{Co}\left(\mathrm{H}_{2} \mathrm{O}\right)_{6}\right] \mathrm{Cl}_{2}$, rosa, cores decorrentes da presença dos complexos tetraclorocobaltato(II) e hexaaquacobalto(II), de geometrias respectivamente tetraédrica e octaédrica ${ }^{9}$.

Antes da execução do experimento, quando indagados sobre possíveis produtos da decomposição do bicarbonato de sódio, os alunos geralmente propõem $\mathrm{CO}_{2}$ e $\mathrm{H}_{2} \mathrm{O}$. A identidade do produto sólido, entretanto, é um grande desafio. Os alunos tendem a sugerir a formação de $\mathrm{NaOH}$ ou $\mathrm{Na}_{2} \mathrm{O}$, possivelmente por analogia com a bem conhecida obtenção da cal $(\mathrm{CaO})$ a partir do carbonato de cálcio. Não lhes parece natural que seja produzido um carbonato. Por esta razão, é crucial para o experimento o teste de identificação do sólido, feito pela reação com ácido clorídrico, na qual há liberação de $\mathrm{CO}_{2}$. Mesmo assim, muitos alunos atribuem, a princípio, este resultado a uma decomposição incompleta do bicarbonato.

Neste ponto, o estudo quantitativo efetuado, se bem conduzido, fornece os dados decisivos para a formulação correta da equação da reação, como segue:

$$
2 \mathrm{NaHCO}_{3}(\mathrm{~s}) \rightarrow \mathrm{Na}_{2} \mathrm{CO}_{3}(\mathrm{~s})+\mathrm{CO}_{2}(\mathrm{~g})+\mathrm{H}_{2} \mathrm{O}(\mathrm{g})
$$

O bicarbonato de sódio sólido, por sofrer decomposição a temperaturas relativamente baixas (cerca de $100{ }^{\circ} \mathrm{C}$ ), pode atuar como "fermento químico" na preparação de pães e bolos ${ }^{10}$. Acima de $140{ }^{\circ} \mathrm{C}$, a reação é mais rápida. Como libera gás e é endotérmica, a reação é utilizada na produção de espumas termoplásticas estruturais e também como pó químico em extintores de incêndio ${ }^{11}$.

Procurando verificar a possibilidade de uso de um produto comercial no experimento, foram efetuados testes com bicarbonato de sódio p.a. da marca Carlo Erba e dois produtos adquiridos em farmácia, de procedências ICAR e LBS, utilizando uma balança Quimis BG-440, com legibilidade de até $0,001 \mathrm{~g}$. Os resultados de perda de massa, apresentados na Tabela 1, demonstram que os três reagentes podem ser usados.

Tabela 1. Porcentagens de perda de massa de amostras de bicarbonato de sódio de três procedências

\begin{tabular}{cccc}
\hline Ensaio & ICAR & LBS & Carlo Erba \\
\hline 1 & 36,74 & 36,84 & 37,09 \\
2 & 36,89 & 36,93 & 36,97 \\
3 & 36,62 & 36,90 & 36,78 \\
Média $^{a}$ & 36,75 & 36,89 & 36,95 \\
\hline
\end{tabular}

${ }^{\mathrm{a}}$ perda de massa esperada $=36,92 \%$

O produto ICAR foi utilizado em uma disciplina de Química Geral para alunos do primeiro ano do curso de Farmácia-Bioquímica da Faculdade de Ciências Farmacêuticas, USP e uma disciplina de Química Inorgânica para alunos do segundo ano do curso de Engenharia - Grande Área Química, da Escola Politécnica, USP, em 2002. A Figura 1 resume os resultados obtidos.

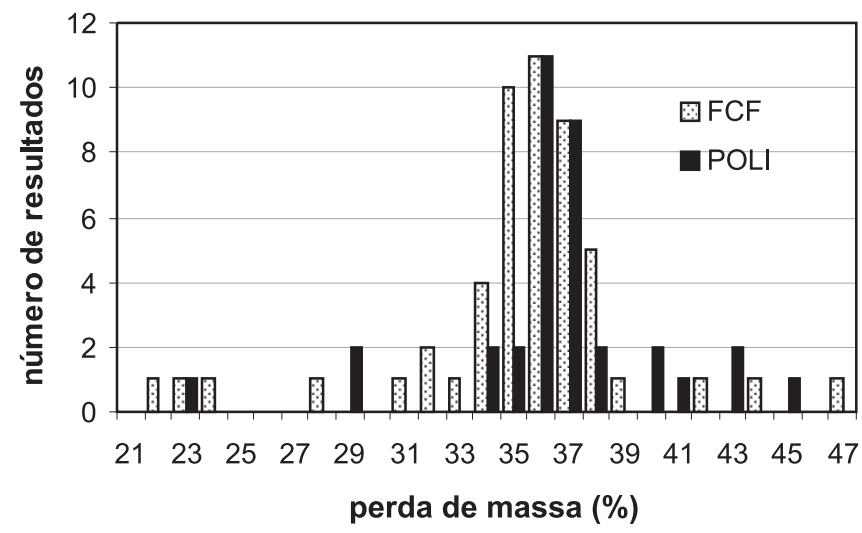

Figura 1. Resultados de perda de massa das amostras de $\mathrm{NaHCO}_{3}$ obtidos pelos alunos dos cursos de Farmácia-Bioquímica (Faculdade de Ciências Farmacêuticas, USP) e de Engenharia - Grande Área Química (Escola Politécnica, USP), 2002. O valor de perda de massa esperado é 36,92\%

Os resultados de perda de massa obtidos pelos alunos da Faculdade de Ciências Farmacêuticas (média de 50 valores $=36,1 \%$ ) tenderam a ser inferiores ao valor esperado $(36,92 \%)$, o que não ocorreu com os alunos da Escola Politécnica (média de 35 valores = $37,0 \%$ ), indicando provavelmente um aquecimento insuficiente no primeiro caso.

A análise das fontes de erro constitui um proveitoso exercício de raciocínio. Podem ser considerados os efeitos, sobre a massa de produto sólido obtida, de fatores como: decomposição incompleta, presença de umidade no bicarbonato de partida, presença de impureza termoestável no bicarbonato, absorção de umidade pelo produto sólido, etc. São fontes de erros sistemáticos, que vão afetar o resultado numa dada direção, contrariamente aos erros aleatórios que os alunos costumam mencionar vagamente nos seus relatórios.

Já a pergunta "Por que o resíduo é um carbonato e não um óxido?" admite respostas sob diferentes enfoques e vários graus de profundidade, dependendo dos objetivos da disciplina e de sua posição na grade curricular. Pode-se discutir a influência das energias reticulares dos sólidos envolvidos, os conceitos ácido-base, com destaque para a teoria de Lux-Flood, ou ainda a variação da energia livre em função da temperatura, representada sob a forma de diagramas tipo Ellingham. Estas três abordagens são apresentadas a seguir.

\section{Energias reticulares dos óxidos metálicos}

Os óxidos normais dos metais mais eletropositivos (grupos 1 e 2 e lantanídeos) são compostos tipicamente iônicos, constituídos pelo cátion metálico e o ânion $\mathrm{O}^{2-}$. A formação de um óxido iônico envolve várias etapas que requerem energia, necessária para dissociar a molécula de $\mathrm{O}_{2}\left(496 \mathrm{~kJ} \mathrm{~mol}^{-1}\right)$, formar o ânion $\mathrm{O}^{2-}$ (a afinidade eletrônica do átomo de oxigênio liberta $141 \mathrm{~kJ} \mathrm{~mol}^{-1}$, na $1^{\mathrm{a}}$ etapa, porém na $2^{\mathrm{a}}$ consome $844 \mathrm{~kJ} \mathrm{~mol}^{-1}$ ) e formar o cátion metálico a partir do metal. Os óxidos iônicos devem, portanto, apresentar uma energia reticular elevada, para compensar esse grande consumo de energia ${ }^{12}$.

As energias reticulares dependem, entre outros fatores, das cargas dos íons e das distâncias entre eles. A Tabela 2 ilustra o efeito da maior carga dos cátions alcalino-terrosos, comparativamente aos alcalinos. Nota-se também que as energias reticulares, numa família, variam menos nos carbonatos do que nos óxidos. Isto ocorre porque a soma dos raios do cátion e do ânion nos carbonatos é dominada pelo maior tamanho do íon $\mathrm{CO}_{3}{ }^{2-}$, com 185 pm de raio ${ }^{13}$. Já nos óxidos, por ser o raio do íon $\mathrm{O}^{2-}, 140$ pm, comparável ao dos cátions, a energia reticular é mais sensível ao tamanho dos cátions ${ }^{14}$. 
Tabela 2. Influência dos raios catiônicos do metal $\mathrm{M}$ nas energias reticulares, $U$, de óxidos e carbonatos ${ }^{\mathrm{a}}$

\begin{tabular}{lrrrrrr}
\hline & $\mathrm{Na}$ & $\mathrm{K}$ & $\mathrm{Cs}$ & $\mathrm{Mg}$ & $\mathrm{Ca}$ & $\mathrm{Ba}$ \\
\hline Raio do cátion $(\mathrm{pm})$ & 95 & 133 & 169 & 72 & 100 & -35 \\
$U_{\mathrm{MxO}}\left(\mathrm{kJ} \mathrm{mol}^{-1}\right)$ & -2724 & -2427 & -2205 & -3923 & -3517 & -3120 \\
$U_{\mathrm{MxC3}}\left(\mathrm{kJ} \mathrm{mol}^{-1}\right)$ & -2300 & -2084 & -1920 & -3178 & -2986 & -2614 \\
$U_{\mathrm{MxCO} 3}-U_{\mathrm{MxO}}\left(\mathrm{kJ} \mathrm{mol}^{-1}\right)$ & 424 & 343 & 285 & 745 & 531 & 506 \\
\hline
\end{tabular}

a dados das refs. 1 e 15

As estabilidades térmicas dos carbonatos podem ser explicadas com base nos valores relativos das energias reticulares. Usando um modelo simplificado, o processo:

$\mathrm{M}_{\mathrm{x}} \mathrm{O}(\mathrm{s})+\mathrm{CO}_{2}(\mathrm{~g}) \rightarrow \mathrm{M}_{\mathrm{x}} \mathrm{CO}_{3}(\mathrm{~s})$

pode ser considerado como a inserção de $\mathrm{CO}_{2}$ no retículo cristalino pré-existente do óxido metálico, provocando uma expansão do mesmo. A energia necessária para a expansão é compensada pela energia liberada pela combinação do íon óxido com o $\mathrm{CO}_{2}$, formando o íon $\mathrm{CO}_{3}{ }^{2-}$. Entretanto, quanto maior, em módulo, a energia reticular do óxido metálico, maior a energia necessária para a expansão do retículo. Conclui-se que a conversão do óxido em carbonato é mais difícil no caso do lítio do que do césio e é também mais difícil para os metais alcalino-terrosos do que para os alcalinos. Conseqüentemente, a decomposição do carbonato, produzindo o óxido e $\mathrm{CO}_{2}$, segue a tendência oposta. Por essa razão, os carbonatos dos metais alcalino-terrosos se decompõem quando aquecidos, enquanto o único dos carbonatos alcalinos que sofre decomposição térmica é o de lítio ${ }^{16}$.

As temperaturas de decomposição dos carbonatos, à pressão de $1 \mathrm{~atm}$, fornecidas pela ref. 17, são $\mathrm{MgCO}_{3} 404{ }^{\circ} \mathrm{C}, \mathrm{CaCO}_{3} 826^{\circ} \mathrm{C}$, $\mathrm{SrCO}_{3} 1098^{\circ} \mathrm{C}, \mathrm{BaCO}_{3} 1370{ }^{\circ} \mathrm{Ce} \mathrm{Li}_{2} \mathrm{CO}_{3} 1157^{\circ} \mathrm{C}$. Esses dados diferem conforme a fonte ${ }^{15,18}$, porém, a tendência de variação é a mesma. $\mathrm{O} \mathrm{BeCO}_{3}$ não foi incluído, pois só ocorre na forma hidratada ${ }^{15}$.

Verifica-se que, quanto mais fortes as ligações no óxido metálico, menor a temperatura de decomposição do carbonato. Para cátions com maior carga e menor raio, a diferença entre as energias reticulares do óxido e do carbonato é maior (vide Tabela 2), favorecendo a conversão carbonato $\rightarrow$ óxido, que ocorre com grande aumento de energia reticular ${ }^{13,19}$.

Os comportamentos discrepantes do lítio e do berílio, que formam cátions muito pequenos, em relação aos demais membros das famílias, servem de exemplo para discutir a influência do poder polarizante dos cátions ${ }^{18,19}$. A decomposição ocorre mais facilmente quando o cátion polariza o íon carbonato, desmembrando-o em íon $\mathrm{O}^{2-}$ e $\mathrm{CO}_{2}$. Fatores que influem no poder polarizante e o caráter parcial covalente das ligações podem também ser abordados, ampliando a comparação com outros sistemas. O efeito dos elétrons $d$, por exemplo, fica patente quando se consideram os carbonatos $\mathrm{CdCO}_{3} \mathrm{e}$ $\mathrm{PbCO}_{3}$ que se decompõem a cerca de $350{ }^{\circ} \mathrm{C}$, embora $\mathrm{Cd}^{2+}{\mathrm{e} \mathrm{Pb}^{2+}}^{2+}$ apresentem raios iônicos semelhantes ao do cátion cálcio ${ }^{18}$.

\section{Teoria ácido-base de Lux-Flood}

Desde os tempos de Berzelius, os óxidos estão vinculados a características ácidas e básicas evidenciadas, entre outras propriedades, pela habilidade de reagirem entre si formando sais ${ }^{20}$. Em 1939, Lux formulou uma definição de ácidos e bases adequada para esse tipo de reações, baseada na Equação $3^{20-22}$.

Base $=$ Ácido $+\mathrm{O}^{2-}$

Esta teoria se assemelha à de Brønsted, porém, ao invés do próton, a entidade transferida é o ânion óxido $\left(\mathrm{O}^{2-}\right)$. Os ácidos atuam como receptores de íons $\mathrm{O}^{2-} \mathrm{e}$ as bases, como doadoras. A teoria é particularmente útil para reações em altas temperaturas, que ocorrem em processos metalúrgicos, cerâmicos e geoquímicos ${ }^{20,22,23}$.

A Equação 2 vista anteriormente é um exemplo genérico de reação de neutralização entre uma base $\left(\mathrm{M}_{\mathrm{x}} \mathrm{O}\right)$ e um ácido $\left(\mathrm{CO}_{2}\right)$. A variação de energia livre dessas reações permite estabelecer uma escala de basicidade dos óxidos, conforme proposto por Flood e Förland ${ }^{17,20}$. Os óxidos de metais alcalinos são em geral bases mais fortes do que os de alcalino-terrosos e, em cada grupo, a basicidade do óxido aumenta com o tamanho do metal ${ }^{15,17}$. Isto se justifica porque a interação do cátion $\mathrm{M}^{+}$com o íon $\mathrm{O}^{2-}$ é mais fraca do que no caso de um cátion $\mathrm{M}^{2+}$ e também é mais fraca para cátions maiores, resultando um óxido com maior facilidade de ceder o íon $\mathrm{O}^{2-}$ (Equação 3) para um óxido ácido ${ }^{17}$.

A ordem de caráter ácido-básico de alguns óxidos é mostrada abaixo $^{12}$ :

$$
\underset{\text { mais básico }}{\stackrel{\mathrm{K}_{2} \mathrm{O}, \mathrm{Na}_{2} \mathrm{O}, \mathrm{BaO}, \mathrm{CaO}, \mathrm{MgO}, \mathrm{CuO}, \mathrm{H}_{2} \mathrm{O}, \mathrm{SiO}_{2}, \mathrm{CO}_{2}, \mathrm{~N}_{2} \mathrm{O}_{5}, \mathrm{SO}_{3}}{\longrightarrow}}
$$

Quanto mais afastados estiverem dois óxidos nessa série, mais estável será o composto formado quando os óxidos reagirem entre $\mathrm{si}^{12}$, conforme atestam os valores de variação de energia livre padrão, fornecidos na Tabela 3 .

Tabela 3. Valores das variações de entalpia padrão e de energia livre padrão, a $25^{\circ} \mathrm{C}$, para reações entre óxidos alcalinos e alcalino-terrosos e anidridos ácidos ${ }^{24,25}$

\begin{tabular}{lcc}
\hline Reação & $\Delta_{\mathrm{r}} H^{\theta}\left(\mathrm{kJ} \mathrm{mol}^{-1}\right)$ & $\Delta_{\mathrm{r}} G^{\theta}\left(\mathrm{kJ} \mathrm{mol}^{-1}\right)$ \\
\hline $\mathrm{Na}_{2} \mathrm{O}(\mathrm{s})+\mathrm{CO}_{2}(\mathrm{~g}) \rightarrow \mathrm{Na}_{2} \mathrm{CO}_{3}(\mathrm{~s})$ & -319 & -274 \\
$\mathrm{~K}_{2} \mathrm{O}(\mathrm{s})+\mathrm{CO}_{2}(\mathrm{~g}) \rightarrow \mathrm{K}_{2} \mathrm{CO}_{3}(\mathrm{~s})$ & -393 & -348 \\
$\mathrm{MgO}(\mathrm{s})+\mathrm{CO}_{2}(\mathrm{~g}) \rightarrow \mathrm{MgCO}_{3}(\mathrm{~s})$ & -117 & -65 \\
$\mathrm{CaO}(\mathrm{s})+\mathrm{CO}_{2}(\mathrm{~g}) \rightarrow \mathrm{CaCO}_{3}(\mathrm{~s})$ & -178 & -130 \\
$\mathrm{BaO}(\mathrm{s})+\mathrm{CO}_{2}(\mathrm{~g}) \rightarrow \mathrm{BaCO}_{3}(\mathrm{~s})$ & -267 & -216 \\
$\mathrm{CaO}(\mathrm{s})+\mathrm{SO}_{3}(\mathrm{~g}) \rightarrow \mathrm{CaSO}_{4}(\mathrm{~s})$ & -402 & -345 \\
\hline
\end{tabular}

Os metais alcalinos formam bases muito fortes e oxossais muito estáveis ${ }^{10}$. Já os óxidos alcalino-terrosos, menos básicos, formam oxossais menos estáveis ao calor, incluindo-se aí os carbonatos ${ }^{15}$.

\section{Diagrama tipo Ellingham}

A decomposição de um carbonato alcalino-terroso, representada pela Equação 4:

$\mathrm{CaCO}_{3}(\mathrm{~s}) \rightarrow \mathrm{CaO}(\mathrm{s})+\mathrm{CO}_{2}(\mathrm{~g})$

é um exemplo comum, nos livros texto, de reação que tende a ocor- 
rer num sentido ou no outro, dependendo da temperatura ${ }^{26-29}$.

O processo inverso é a reação representada genericamente pela Equação 2, que ocorre com consumo de gás, implicando em diminuição de entropia. Se o processo da Equação 2 for termodinamicamente viável, ele deve libertar calor, ou seja, a reação é entalpicamente dirigida. Alguns valores de variação de entalpia estão reunidos na Tabela 3.

Na expressão da variação de energia livre de uma reação, a uma dada temperatura e pressão, o termo entrópico é multiplicado pela temperatura absoluta:

$\Delta_{\mathrm{r}} G^{\theta}=\Delta_{\mathrm{r}} H^{\theta}-T \Delta_{\mathrm{r}} S^{\theta}$

Um carbonato estável à temperatura ambiente poderá, portanto, se decompor quando aquecido, desde que o termo $T \Delta_{\mathrm{r}} S$ sobrepuje o termo $\Delta_{\mathrm{r}} H$, em módulo.

Uma representação gráfica adequada para analisar estes processos é apresentada na Figura 2 e mostra como varia, em função da temperatura, a energia livre da reação de obtenção dos carbonatos a partir dos óxidos correspondentes. Tal diagrama é análogo aos propostos por Ellingham para a energia livre de certas classes de reações $^{30}$, sendo o diagrama mais conhecido o referente à formação de óxidos, muito importante em metalurgia ${ }^{28,31-35}$.

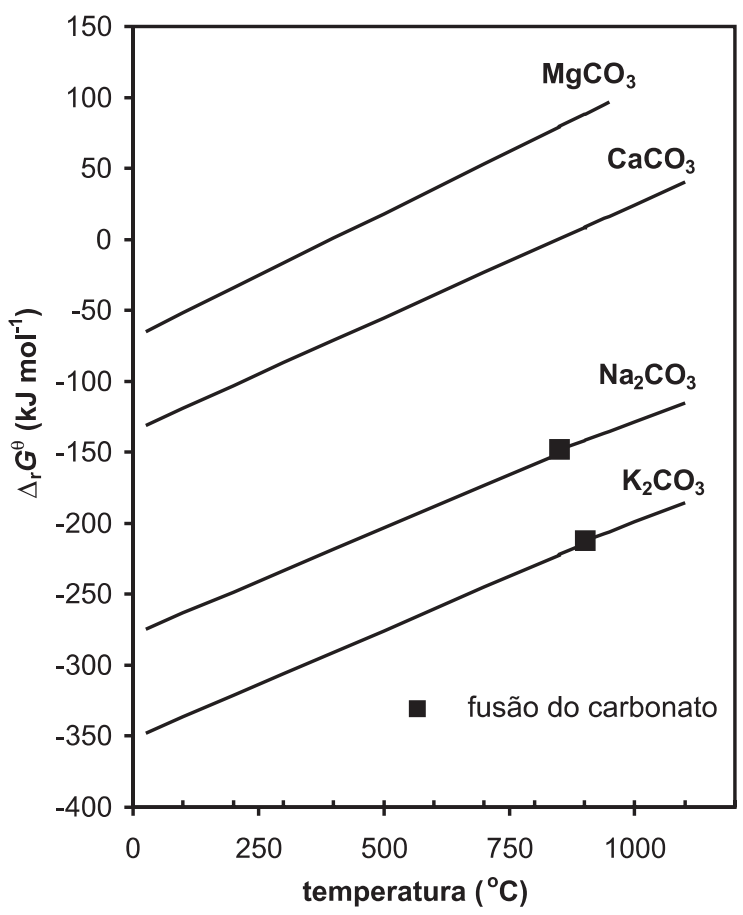

Figura 2. Variação da energia livre padrão, em função da temperatura, da reação $\mathrm{M}_{x} \mathrm{O}+\mathrm{CO}_{2} \rightarrow \mathrm{M}_{x} \mathrm{CO}_{3}$

Para construir a Figura 2, os valores de $\Delta_{\mathrm{r}} H^{\theta}$ e $\Delta_{\mathrm{r}} S^{\theta}$ para a reação representada pela Equação 2 foram calculados utilizando, nas Equações 6 e 7, dados de entalpia padrão de formação, $\Delta_{\mathrm{f}} H^{\theta}$, e de entropia padrão, $S^{\theta}$, dos óxidos, carbonatos e gás carbônico, fornecidos na literatura ${ }^{24,25}$, a $25^{\circ} \mathrm{C}$ :

$\Delta_{\mathrm{r}} H^{\theta}=\Delta_{\mathrm{f}} H^{\theta}{ }_{\mathrm{MxCO} 3}-\Delta_{\mathrm{f}} H^{\theta}{ }_{\mathrm{MxO}}-\Delta_{\mathrm{f}} H^{\theta}{ }_{\mathrm{CO} 2}$

$\Delta_{\mathrm{r}} S^{\theta}=S_{\mathrm{MxCO} 3}^{\theta}-S^{\theta}{ }_{\mathrm{MxO}}-S^{\theta}{ }_{\mathrm{CO} 2}$

A partir dos valores de $\Delta_{\mathrm{r}} H^{\theta}$ e $\Delta_{\mathrm{r}} S^{\theta}$ assim obtidos, calcularam-se os valores de $\Delta_{\mathrm{r}} G^{\theta}$ a diversas temperaturas, pela Equação 5. Como $\Delta_{\mathrm{r}} H^{\theta}$ e $\Delta_{\mathrm{r}} S^{\theta}$ podem ser considerados aproximadamente constantes em amplos intervalos de temperaturas ${ }^{17,26,35,36}$, desde que não ocorram mudanças de fase das substâncias, o gráfico de $\Delta_{\mathrm{r}} G^{\theta}$ vs $T$ apresenta trechos de reta, cujo coeficiente angular é $-\Delta_{\mathrm{r}} S^{\theta}$ e coeficiente linear, $\Delta_{\mathrm{r}} H^{\theta}$.

As retas da Figura 2 são ascendentes porque representam processos que ocorrem com diminuição de entropia (Equação 2). Observa-se que, no caso do magnésio e do cálcio, $\Delta_{\mathrm{r}} G^{\theta}$ se torna positivo a partir de 396 e $848^{\circ} \mathrm{C}$, respectivamente. Isto significa que, do ponto de vista termodinâmico, abaixo dessas temperaturas os carbonatos são estáveis e acima delas, eles se decompõem. Já os carbonatos de sódio e potássio são estáveis em todo o intervalo de temperaturas da Figura 2. Um diagrama mais completo, incluindo outros carbonatos, pode ser encontrado na ref. 31 .

Em disciplinas mais avançadas ou mais específicas, o gráfico da Figura 2 pode ser construído levando em conta a variação de $\Delta_{\mathrm{f}} H^{\theta} \mathrm{e}$ $S^{\theta}$ com a temperatura. As equações necessárias podem ser deduzidas a partir de polinômios que fornecem as capacidades caloríficas molares à pressão constante, $C_{p}$, em função da temperatura absoluta $T$ e de parâmetros $a, b, c, d$ e $e$, tabelados na literatura ${ }^{24,25,34}$, para as diferentes substâncias:

$C_{p}=a+b T+c T^{2}+d T^{3}+e T^{-2}$

A título de comparação, foram calculados $\Delta_{\mathrm{r}} H^{\theta}, \Delta_{\mathrm{r}} S^{\theta}$ e $\Delta_{\mathrm{r}} G^{\theta}$ para o caso do carbonato de sódio, utilizando as equações de Shomate (polinômios de $\Delta_{\mathrm{f}} H^{\theta}$ e $S^{\theta}$ em função de $T$ ) para $\mathrm{Na}_{2} \mathrm{O}, \mathrm{CO}_{2}$ e $\mathrm{Na}_{2} \mathrm{CO}_{3}$, fornecidas na ref. 24. A Tabela 4 compara esses resultados com o cálculo mais simplificado de $\Delta_{\mathrm{r}} G^{\theta}$, baseado em valores constantes de $\Delta_{\mathrm{r}} H^{\theta}$ e $\Delta_{\mathrm{r}} S^{\theta}$. Observam-se diferenças nos dois conjuntos de $\Delta_{\mathrm{r}} G^{\theta}$ a partir de $500{ }^{\circ} \mathrm{C}$. Os valores de $\Delta S^{\theta}$ variam mais pronunciadamente com a temperatura do que os de $\Delta_{r} H^{\theta}$, porém há uma compensação parcial entre eles, no cálculo de $\Delta_{\mathrm{r}} G^{\theta}$, por contribuírem com sinais opostos. Essa compensação torna-se menos eficiente com o aumento da temperatura. Efetuando-se extrapolações até o ponto em que $\Delta_{\mathrm{r}} G^{\theta}$ se anula, as temperaturas são bem diferentes, a saber, $1970{ }^{\circ} \mathrm{C}$ pelo cálculo simplificado e $2450{ }^{\circ} \mathrm{C}$, pelo tratamento mais rigoroso. Nenhuma dessas temperaturas pode ser alcançada na chama de um bico de Bünsen, não ocorrendo, portanto, a decomposição do carbonato de sódio.

Tabela 4. Comparação entre dois conjuntos de valores de variação de energia livre padrão da reação $\mathrm{Na}_{2} \mathrm{O}(\mathrm{s})+\mathrm{CO}_{2}(\mathrm{~g}) \rightarrow \mathrm{Na}_{2} \mathrm{CO}_{3}$ (s, até $\left.850{ }^{\circ} \mathrm{C}\right),\left(1\right.$, acima de $\left.850^{\circ} \mathrm{C}\right)$ em função da temperatura

\begin{tabular}{ccccc}
\hline $\begin{array}{c}\text { Temperatura } \\
\left({ }^{\circ} \mathrm{C}\right)\end{array}$ & $\begin{array}{c}\Delta_{\mathrm{r}} G^{\theta \mathrm{a}} \\
\left(\mathrm{kJ} \mathrm{mol}^{-1}\right)\end{array}$ & $\begin{array}{c}\Delta_{\mathrm{r}} H^{\theta \mathrm{b}} \\
\left(\mathrm{kJ} \mathrm{mol}^{-1}\right)\end{array}$ & $\begin{array}{c}\Delta_{\mathrm{r}} S^{\theta \mathrm{b}} \\
\left(\mathrm{J} \mathrm{mol}^{-1} \mathrm{~K}^{-1}\right)\end{array}$ & $\begin{array}{c}\Delta_{\mathrm{r}} G^{\theta \mathrm{c}} \\
\left(\mathrm{kJ} \mathrm{mol}^{-1}\right)\end{array}$ \\
\hline 25 & $-274,5$ & $-319,3$ & $-150,0$ & $-274,5$ \\
100 & $-263,3$ & $-318,8$ & $-148,8$ & $-263,3$ \\
200 & $-248,3$ & $-317,9$ & $-146,6$ & $-248,6$ \\
300 & $-233,3$ & $-316,0$ & $-142,9$ & $-234,1$ \\
500 & $-203,3$ & $-309,1$ & $-132,5$ & $-206,6$ \\
700 & $-173,3$ & $-305,6$ & $-128,6$ & $-180,4$ \\
850 & $-150,8$ & $-302,1$ & $-125,3$ & $-161,3$ \\
900 & $-141,7$ & $-270,6$ & $-97,3$ & $-156,4$ \\
1100 & $-115,2$ & $-275,7$ & $-101,5$ & $-136,3$ \\
\hline
\end{tabular}

a até $850{ }^{\circ} \mathrm{C}: \Delta_{\mathrm{r}} G^{\theta}=-319,27+0,15004 T$; acima de $850{ }^{\circ} \mathrm{C}: \Delta_{\mathrm{r}} G^{\theta}=-$ 297,01+0,13242T; $T=$ temperatura $(\mathrm{K})^{24} ;{ }^{\text {b }}$ calculada pelas equações de Shomate ${ }^{24} ;{ }^{\text {c }}$ calculada pela equação $\Delta_{\mathrm{r}} G^{\theta}=\Delta_{\mathrm{r}} H^{\theta}-T \Delta_{\mathrm{r}} S^{\theta}$ utilizando os valores de $\Delta_{\mathrm{r}} H^{\theta \mathrm{b}}$ e $\Delta_{\mathrm{r}} S^{\theta \mathrm{b}}$ 
Finalmente cabe mencionar que a partir dos valores da grandeza $\Delta_{r} G^{\theta}$ a várias temperaturas, para os processos das Equações 1 e 2, pode-se calcular a constante de equilíbrio termodinâmica padrão e a pressão parcial do gás carbônico a diferentes temperaturas ${ }^{34,35}$.

\section{A PRODUÇÃO DE BARRILHA E O PROCESSO SOLVAY}

O carbonato de sódio anidro é conhecido comercialmente como barrilha ou soda (em inglês, "soda ash"). Historicamente, era produzido evaporando o extrato de cinzas de certas plantas, como a Barilla espanhola ${ }^{37}$, ou barrilheira, planta do litoral de folhas espinhosas ${ }^{38} \mathrm{e}$ as plantas marinhas Chenopodium, Salicornia, Salsola, etc. ${ }^{39}$ Até o final do século XVIII, estas eram as principais fontes de carbonato de sódio ${ }^{33}$

Em 1775, procurando evitar a dependência exterior quanto à importação do carbonato de sódio natural, o governo francês estabeleceu um prêmio a quem propusesse um processo satisfatório de obtenção de $\mathrm{Na}_{2} \mathrm{CO}_{3}$ a partir de $\mathrm{NaCl}^{13,40-42}$. O carbonato era necessário para a indústria têxtil e do vidro e também para a produção de hidróxido de sódio, ou soda cáustica, através da reação com hidróxido de cálcio:

$$
\mathrm{Ca}(\mathrm{OH})_{2}(\mathrm{~s})+\mathrm{Na}_{2} \mathrm{CO}_{3}(\mathrm{aq}) \rightarrow \mathrm{CaCO}_{3}(\mathrm{~s})+2 \mathrm{NaOH}(\mathrm{aq})
$$

$\mathrm{O} \mathrm{NaOH}$ por sua vez se destinava principalmente à fabricação de sabão ${ }^{42}$.

Nicholas Leblanc (1742-1806) concorreu com o processo que leva o seu nome e que se baseia na conversão de $\mathrm{NaCl}$ primeiramente em $\mathrm{Na}_{2} \mathrm{SO}_{4}$, por tratamento com $\mathrm{H}_{2} \mathrm{SO}_{4}$. A seguir o $\mathrm{Na}_{2} \mathrm{SO}_{4}$ é aquecido num forno com carvão e $\mathrm{CaCO}_{3}$, produzindo uma mistura sólida de $\mathrm{Na}_{2} \mathrm{CO}_{3}$ e CaS, da qual o carbonato de sódio é extraído com água $^{42}$. Foi o primeiro processo para produção comercial de soda em grande escala, porém era caro e causava problemas sérios de poluição ambiental ${ }^{33,37,40,43}$.

No final do século XIX, o processo Leblanc encontrava-se quase totalmente substituído pelo método desenvolvido pelo químico belga Ernest Solvay (1838-1922) ${ }^{42}$. O processo Solvay consiste em bombear $\mathrm{CO}_{2}$ na parte inferior de uma torre, enquanto uma salmoura saturada com amônia é introduzida pelo topo. A reação que ocorre nessa torre, denominada torre de carbonatação, pode ser representada pela equação:

$\mathrm{NaCl}(\mathrm{aq})+\mathrm{NH}_{3}(\mathrm{~g})+\mathrm{CO}_{2}(\mathrm{~g})+\mathrm{H}_{2} \mathrm{O}(\mathrm{l}) \rightarrow \mathrm{NaHCO}_{3}(\mathrm{~s})+\mathrm{NH}_{4} \mathrm{Cl}(\mathrm{aq})$

O bicarbonato de sódio precipita preferencialmente da mistura reacional, em temperaturas abaixo de $15^{\circ} \mathrm{C}$, por ser menos solúvel (solubilidade a $0{ }^{\circ} \mathrm{C}=0,82 \mathrm{~mol} \mathrm{~L}^{-1}$ ) do que as outras combinações possíveis, $\mathrm{NH}_{4} \mathrm{Cl}\left(5,5 \mathrm{~mol} \mathrm{~L}^{-1}\right), \mathrm{NaCl}\left(6,1 \mathrm{~mol} \mathrm{~L}^{-1}\right)$ e $\mathrm{NH}_{4} \mathrm{HCO}_{3}$ $\left(1,5 \mathrm{~mol} \mathrm{~L}^{-1}\right)^{44}$. A presença de íons amônio na mistura faz com que predominem íons bicarbonato, em detrimento dos íons carbonato, que são bases de Brønsted muito fortes.

O sólido que se forma é separado por filtração e contém $\mathrm{NaHCO}_{3}$ (cerca de 76\%), $\mathrm{Na}_{2} \mathrm{CO}_{3}, \mathrm{NH}_{4} \mathrm{HCO}_{3}, \mathrm{NaCl}, \mathrm{NH}_{4} \mathrm{Cl}$ e $\mathrm{H}_{2} \mathrm{O}$. A decomposição térmica deste material libera gás carbônico, amônia e vapor d'água, produzindo carbonato de sódio, cuja principal impureza é o cloreto de sódio, com resultados analíticos da ordem de 99,6\% de $\mathrm{Na}_{2} \mathrm{CO}_{3}$ e $0,15 \%$ de $\mathrm{NaCl}$. Produtos mais puros, para fins analíticos ou alimentícios, podem ser obtidos a partir de uma solução do carbonato de sódio industrial, borbulhando-se gás carbônico para convertê-lo no bicarbonato. Este cristaliza, podendo ser separado. Um produto típico para fins alimentícios apresenta a composição: $99,7 \% \mathrm{NaHCO}_{3}, 0,2 \% \mathrm{Na}_{2} \mathrm{CO}_{3}, 0,004 \% \mathrm{NaCl}, 0,05 \% \mathrm{H}_{2} \mathrm{O}$ e $0,003 \%$ de impurezas insolúveis ${ }^{45}$.
Em 1863, Ernest Solvay fundou, com seu irmão Alfred, a Societé Solvay \& Cie., em Bruxelas. A primeira fábrica, construída em Couillet, na Bélgica ${ }^{46}$, iniciou suas atividades em 1865. As dificuldades técnicas surgidas nos primeiros anos foram resolvidas uma a uma, graças à persistência e engenhosidade de Ernest Solvay, até o processo tornar-se rentável ${ }^{4,47}$.

Solvay foi inovador também em seus métodos, ao estabelecer controles rigorosos a cada estágio da fabricação ${ }^{48}$. Em 1897, diversificou a empresa, instalando a primeira unidade de eletrólise de salmoura $^{49}$. O êxito empresarial permitiu a Solvay criar inúmeras obras sociais e instituições científicas ${ }^{48}$. Foi o patrocinador das Conferências Solvay, a primeira das quais se realizou em 1911, reunindo alguns dos cérebros mais privilegiados da Ciência, conforme se pode ver na famosa foto desse evento ${ }^{50}$.

O processo Solvay foi o primeiro processo industrial econômico funcionando em fluxo contínuo ${ }^{40}$. Constituiu um sucesso de engenharia por utilizar eficientemente a matéria prima, reciclando os subprodutos ${ }^{42,51,52}$. O calcáreo $\left(\mathrm{CaCO}_{3}\right)$ é aquecido para produzir $\mathrm{CO}_{2}$ e $\mathrm{CaO}$, conforme a Equação 4. $\mathrm{O} \mathrm{CO}_{2}$ destina-se à reação representada pela Equação 10, enquanto o $\mathrm{CaO}$ é convertido em $\mathrm{Ca}(\mathrm{OH})_{2}$ por tratamento com água. $\mathrm{O} \mathrm{Ca}(\mathrm{OH})_{2}$ permite converter o subproduto $\mathrm{NH}_{4} \mathrm{Cl}$ (ver Equação 10) em $\mathrm{NH}_{3}$ reutilizado para saturar desse gás a salmoura que alimenta a torre de carbonatação. A regeneração da amônia, representada pela Equação 11, torna o processo economicamente viável, por ser a amônia um reagente relativamente caro.

$\mathrm{CaO}(\mathrm{s})+2 \mathrm{NH}_{4} \mathrm{Cl}(\mathrm{aq}) \rightarrow 2 \mathrm{NH}_{3}(\mathrm{~g})+\mathrm{CaCl}_{2}(\mathrm{aq})+\mathrm{H}_{2} \mathrm{O}(\mathrm{l})$

$\mathrm{O} \mathrm{CO}_{2}$ produzido na decomposição térmica do bicarbonato de sódio (Equação 1) também pode ser reaproveitado no processo.

O resultado global de todas essas etapas é a reação entre o calcário e o sal, resumida pela Equação 12, que é termodinamicamente impossível de ocorrer numa só etapa ${ }^{5}$.

$2 \mathrm{NaCl}(\mathrm{aq})+\mathrm{CaCO}_{3}(\mathrm{~s}) \rightarrow \mathrm{Na}_{2} \mathrm{CO}_{3}(\mathrm{~s})+\mathrm{CaCl}_{2}(\mathrm{aq})$

Para produzir uma tonelada de $\mathrm{Na}_{2} \mathrm{CO}_{3}$, são consumidos $1,5 \mathrm{t}$ de salmoura, 1,5 t de calcário, 50,0 t de água e apenas $2 \mathrm{~kg}$ de amônia. Grande parte da água é utilizada para a refrigeração da torre de carbonatação ${ }^{53}$.

$\mathrm{O}$ único subproduto do processo Solvay é, portanto, o $\mathrm{CaCl}_{2}$. A demanda para esta substância é, porém, limitada. Os principais usos são como agente desidratante e para descongelar neve em ruas e estradas, em países de clima frio ${ }^{37,44,51}$.

No passado, muito cloreto de cálcio foi despejado em lagos e rios, até que legislações de proteção ao meio ambiente proibissem tal procedimento. Em parte por esta razão e em parte pela existência de fontes naturais abundantes de trona em Wyoming, o processo Solvay é atualmente obsoleto nos Estados Unidos ${ }^{5,42}$.

Os depósitos de trona, $\mathrm{Na}_{2} \mathrm{CO}_{3} \cdot \mathrm{NaHCO}_{3} \cdot 2 \mathrm{H}_{2} \mathrm{O}$, descobertos em Green River, Wyoming, EUA, em 1938, foram utilizados para a produção de barrilha a partir de 1953. Estima-se que sejam suficientes para suprir a demanda mundial por cerca de $2000 \operatorname{anos}^{37}$. É possível que essa fonte venha a substituir o processo Solvay, por ser mais barata e pelos inconvenientes causados pelo sub-produto deste, o cloreto de cálcio ${ }^{44,54}$.

Em vários lugares do mundo, o processo Solvay continua, entretanto, em uso, sendo responsável por cerca de $70 \%$ do suprimento mundial de barrilha ${ }^{5}$. O grupo Solvay tem oito fábricas européias que ainda utilizam esse processo ${ }^{55}$. No Brasil, o processo é utilizado, para produzir barrilha a partir do sal marinho e do calcáreo, pela empresa Álcalis, em Arraial do Cabo, no Rio de Janeiro, que produz até $220 \mathrm{mil} \mathrm{t/ano}{ }^{56}$. 
Em 1999, a indústria química brasileira importou 352 t de barrilha, enquanto as vendas internas foram de $207 \mathrm{t}$, cujo destino aproximado está representado no diagrama da Figura $3^{57}$. Devido aos inúmeros e fundamentais processos que empregam o carbonato de sódio como matéria prima, ele é o nono produto químico inorgânico mais importante, em termos de quantidade utilizada 5 .

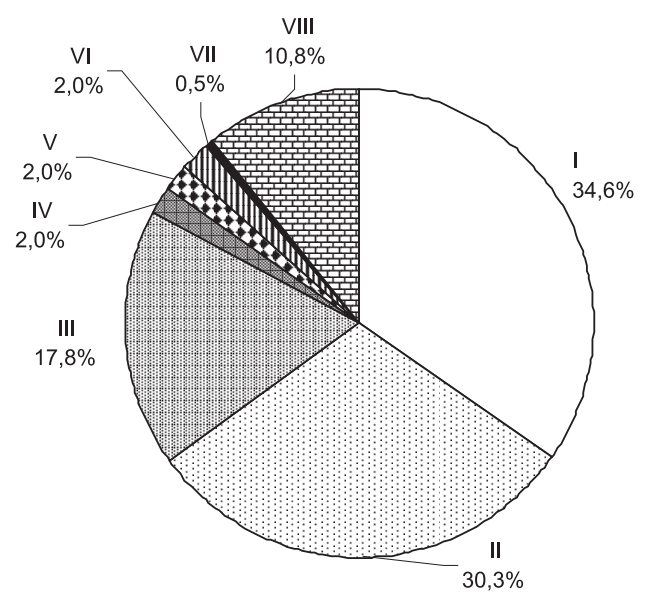

Figura 3. Destino aproximado das vendas internas de $\mathrm{Na}_{2} \mathrm{CO}_{3}$ da empresa Álcalis em 2000: $I$ = sabões e detergentes; $I I=$ vidros; $I I I=$ processos químicos; $I V=$ couro $; V=$ alumínio; $V I=$ tratamento de água $; V I I=$ papel e celulose; VIII = revenda. Dados obtidos da ref. 57

Uma rota alternativa para a produção de bicarbonato de sódio, que pode ser atraente conforme os preços do mercado, utiliza gás carbônico, sub-produto de diversos processos, e hidróxido de sódio, obtido pela eletrólise da salmoura e cuja demanda é geralmente inferior à do cloro. Este processo é utilizado pela empresa Química Geral do Nordeste S.A., integrante do pólo industrial de Camaçari ${ }^{58}$.

\section{CONCLUSÕES}

O experimento proposto utilizando bicarbonato de sódio comercial tem objetivos qualitativos (deduzir a equação do processo, com base na identificação dos produtos) e quantitativos (efetuar cálculos estequiométricos a partir da massa inicial do bicarbonato). Não se limita a isso, porém. O experimento é estimulante para os alunos, que se inclinam a esperar um óxido como produto da decomposição térmica e se surpreendem ao identificá-lo como carbonato. Neste trabalho, são sugeridos conceitos que podem ser explorados na discussão do resultado, em diferentes níveis de profundidade.

A reação estudada é a última etapa do processo Solvay de obtenção da soda, um processo de importância histórica e comercial, no qual as reações ácido-base desempenham um importante papel. $\mathrm{Cu}$ riosamente, o processo Solvay beneficia-se tanto da estabilidade térmica do carbonato de sódio, o seu produto final, quanto da facilidade de decomposição do carbonato de cálcio, uma das suas matérias primas.

\section{AGRADECIMENTOS}

As autoras agradecem à Profa. W. Oliveira, pelo apoio e incentivo, e aos alunos que cursaram as disciplinas QFL-137 e QFL-2129, no Instituto de Química, USP, em 2002. A. S. Maia agradece à FAPESP e ao Programa de Aperfeiçoamento de Ensino, PAE, da Pró-Reitoria de Pós-Graduação da USP.

\section{REFERÊNCIAS}

1. Giesbrecht, E., coord.; Experiências de Química, Ed. Moderna/EDUSP: São Paulo, 1979.

2. Maia, A. de S.; Osório, V. K. L.; Resumos da 25a Reunião Anual da Sociedade Brasileira de Química, Poços de Caldas, Brasil, 2002.

3. Shreve, R. N.; Brink Jr., J. A.; Indústrias de Processos Químicos, $4^{\mathrm{a}}$ ed., Ed. Guanabara: Rio de Janeiro, 1980, cap. 13, p. 185; Selinger, B.; Chemistry in the Marketplace, $5^{\text {th }}$ ed., Harcourt Brace \& Co.: Sydney, 1998, p. 240.

4. de Araújo, A. L.; Neves, C. A.; Ferreira, A. M. C.; Araki, K.; Quim. Nova 1998, 21,114

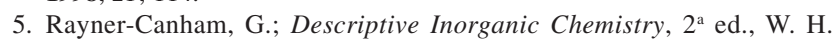
Freeman and Co.: New York, 2000, p. 198

6. Baccan, N.; Godinho, O. E. S.; Aleixo, L. M.; Stein, E.; Introdução à Semimicroanálise Qualitativa, $5^{\text {a }}$ ed., Ed. Unicamp: Campinas, 1994, p. 229

7. Ford, L. A.; Magia Química, Editorial Diana: México, 1982, p. 174.

8. Lee, J. D.; Química Inorgânica não tão concisa, Ed. E. Blücher: São Paulo, 1999, p. 400.

9. Cotton, F. A.; Wilkinson, G.; Advanced Inorganic Chemistry, $4^{\mathrm{a}}$ ed., John Wiley \& Sons: New York, 1980, p. 770; Shakhashiri, B. Z.; Chemical Demonstrations - A Handbook for Teachers of Chemistry, The University of Wisconsin Press: Madison, 1983, vol. 1, p. 283; Kettle, S. F. A.; Physical Inorganic Chemistry, Oxford University Press: Oxford, 1998, p. 175; Ibidem ref. 8, p. 491.

10. Ibidem ref. 8 , cap. 9 .

11. http://www.solvaybicar.com, acessada em Julho 2002.

12. Ibidem ref. 8, p. 276.

13. Mingos, D. M. P.; Essential Trends in Inorganic Chemistry, Oxford University Press: Oxford, 1998, p. 69.

14. Mahan, B. M.; Myers, R. J.; Química, um curso universitário, Ed. E. Blücher Ltda.: São Paulo, 1993, p. 379.

15. Ibidem ref. 8, cap. 11.

16. Wilson, D. E.; Essential Ideas in Inorganic Chemistry, Hodder and Stoughton: London, 1983, p. 25.

17. Brown, G. I.; Introduction to Inorganic Chemistry, $2^{\text {a }}$ ed., Longman Group Ltd.: Essex, 1985, p. 160-161.

18. Huheey, J. E.; Keiter, E. A.; Keiter, R. L.; Inorganic Chemistry - Principles of Structure and Reactivity, $4^{\text {th }}$ ed., Harper Collins: New York, 1993, p. 133.

19. Massey, A. G.; Main Group Chemistry, Ellis Horwood: New York, 1990, p. 130.

20. Flood, H.; Förland, T.; Acta Chem. Scand. 1947, 1, 592.

21. Lux, H.; Z. Elektrochem. 1939, 45, 303 apud ref. 20.

22. Douglas, B.; McDaniel, D.; Alexander, J.; Concepts and Models of Inorganic Chemistry, $3^{\text {rd }}$ ed., John Wiley \& Sons: New York, 1994, p. 333.

23. Flood, H.; Förland, T.; Roald, B.; Acta Chem. Scand. 1947, 1, 790; Jensen, W. B.; The Lewis Acid-base Concepts, John Wiley \& Sons: New York, 1980, p. 54; Chagas, A. P.; Quim. Nova 2000, 23, 126; Koeller, S. L.; Combes, R. L.; Quim. Nova 2002, 25, 226.

24. http://webbook.nist.gov, acessada em Julho 2002.

25. CRC Handbook of Chemistry and Physics, Weast, R. C. , ed.; $69^{\text {th }}$ ed., CRC Press: Boca Raton, 1988-1989.

26. Quagliano, J. V.; Vallarino, L. M.; Química, $3^{\mathrm{a}}$ ed., Ed. Guanabara Dois: Rio de Janeiro, 1979, p. 382.

27. Kotz, J. C.; Purcell, D. F.; Chemistry and Chemical Reactivity, Saunders College Publ.: Fort Worth, 1987, p. 686.

28. Freemantle, M.; Chemistry in Action, MacMillan Education Ltd.: London, 1989, p. 179.

29. Masterton, W. L.; Slowinski, E. J.; Stanitski, C. L.; Princípios de Química, $6^{a}$ ed., LTC - Livros Técnicos e Científicos Ed.: Rio de Janeiro, 1990, cap. 14.

30. Ellingham, H. J. T.; J. Chem. Soc. Ind. Lond. 1944, 63, 125 apud ref. 8, p. 97; Dannatt, C. W.; Ellingham, H. J. T.; Disc. Faraday Soc. 1948, 4, 126.

31. Rosenqvist, T.; Principles of Extractive Metallurgy, McGraw-Hill: New York, 1974, p. 531.

32. Dasent, W. E.; Inorganic Energetics, $2^{\mathrm{a}}$ ed., Cambridge University Press: Cambridge, 1982, p. 28; Greenwood, N. N.; Earnshaw, A.; Chemistry of the Elements, Pergamon Press: Oxford, 1984, p. 327; Fergusson, J. E.; Inorganic Chemistry and the Earth, Pergamon Press: Oxford, 1985, p. 83; Shriver, D. F.; Atkins, P. W.; Langford, G. H.; Inorganic Chemistry, Oxford University Press: Oxford, 1990, p. 229; Ibidem ref. 5, p. 170; Ibidem ref. 8, p. 93.

33. Ibidem ref. 17, p. 111

34. Warn, J. R. W.; Concise Chemical Thermodynamics, Van Nostrand Rheinhold Co. Ltd.: London, 1988, cap. 10. 
35. Chagas, A. P.; Termodinâmica Química, Ed. Unicamp: Campinas, 1999, cap. 10.

36. Pimentel, G. C.; Spratley, R. D.; Química, um Tratamento Moderno, Ed. E. Blücher/EDUSP: São Paulo, 1974, vol. 1, p. 299.

37. Rauh, F. Em Kirk-Othmer Encyclopedia of Chemical Technology; Kroschwitz, J. I.; Howe-Grant, M., eds.; $4^{\text {th }}$ ed., John Wiley \& Sons: New York, 1991, vol. 1, p. 1025.

38. Koogan, A.; Houaiss, A.; Enciclopédia e Dicionário Ilustrado, Edições Delta: Rio de Janeiro, 1995.

39. Partington, J. R.; Química General e Inorgánica, Ed. Dossat: Madrid, 1950, p. 378.

40. Oxtoby, D. W.; Nachtrieb, N. H.; Freeman, W. A.; Chemistry, Science of Change, Saunders College Publ.: Philadelphia, 1990, cap. 24.

41. Hudson, J.; The History of Chemistry, MacMillan: London, 1994, p. 247; Maar, J. H.; Pequena História da Química, Ed. Papa-Livros: Florianópolis, 1999 , p. 681.

42. Hill, J. W.; Petrucci, R. H.; General Chemistry, Instructor's Edition, Prentice Hall: New Jersey, 1996, p. 287.

43. de Moura, A. F.; Quim. Nova 2000, 23, 851.

44. Masterton, W. L.; Slowinski, E. J.; Química Geral Superior, $4^{\text {a }}$ ed., Ed. Interamericana Ltda.: Rio de Janeiro, 1978, p. 400-403.

45. Thieme, C.; Industrial Inorganic Chemicals and Products - An Ullmanns' Encyclopedia, Wiley-VCH: Weinheim, 1998, vol. 5, p. 4389.
46. http://www.solvayindupa.com.br, acessada em Julho 2002.

47. Bensaude-Vincent, B.; Stengers, I.; História da Química, Instituto Piaget: Lisboa, 1992, p. 240; http://www.solvay.com/overview/over3.htm, acessada em Julho 2002.

48. http://www.wallonie-en-ligne.net/1995_Cent_Wallons/Solvay_Ernest.htm, acessada em Outubro 2002.

49. O Cloro, publicação do Setor Álcalis, Solvay S.A., sem data.

50. http://solvayins.ulb.ac.be/fixed/Reference2.html, acessada em Julho 2002; Danon, J.; Quim. Nova 1978, 1, 16.

51. Ibidem ref. 27, p. 761.

52. Ebbing, D. D.; Química Geral, $5^{\text {a }}$ ed., Livros Técnicos e Científicos Ed.: Rio de Janeiro, 1998, vol. II, p. 349.

53. Ibidem ref. 28, p. 477.

54. Glanville, J.; Rau, E.; J. Chem. Educ. 1973, 50, 64; Büchner, W.; Schliebs, R.; Winter, G.; Büchel, K. H.; Industrial Inorganic Chemistry, VCH Publishers: New York, 1989, p. 219.

55. http://www.solvay.com; http://www.sodaash.com, acessadas em Julho 2002

56. http://www.alcalis.com.br, acessada em Junho 2002.

57. Anuário da Indústria Química Brasileira; ABIQUIM, São Paulo, 2000, ano 27, p. 277.

58. http://www.qgn-carbonor.com.br, acessada em Outubro 2002. 